were able to take into account Coulomb repulsion between electrons, leading to corrections for electron-electron scattering. This led to a theory for the negative value of the coefficient of magnetoresistance in disordered metals - a phenomenon which has puzzled physicists for more that 30 years. The same reasoning also gave a long-sought explanation for the zero-voltage-anomaly in tunnel junctions, and predicted the unusual temperature and magnetic field dependencies of resistivity and the Hall effect in twodimensional electron gases and in thin metallic films.

In addition, it was demonstrated that interference phenomena are particularly interesting if magnetic fields are present. Owing to the Bohm-Aharanov effect, the magnetic flux through a closed loop introduces changes in the interference pattern which are periodic in the magnetic field. This leads to the famous and remarkable prediction of the oscillatory behaviour of the resistance of thin hollow cylinders of a normal metal in a field parallel to the axis of the cylinder; it was confirmed beautifully by the spectacular SharvinSharvin experiment (see figure). This work, in effect, opened up in a very fundamental way a new branch of physics which we now call mesoscopics.

The complete calculations of quantum corrections to electrical conductivity and other

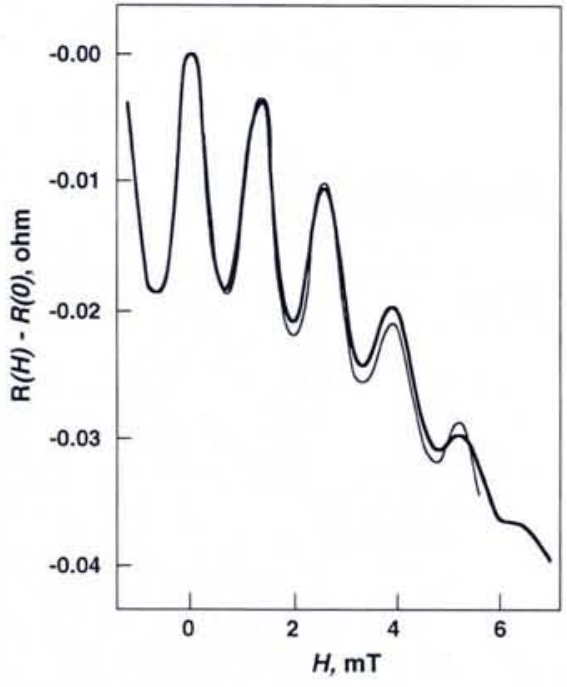

physical characteristics of disordered metals are usually performed using the powerful methods of quantum fieid theory, as pioneered by the Russian school. But the prizewinners also introduced very simple and very elegant reasoning which make it possible to obtain order-of-magnitude estimates of the corrections, and to give a very clear physical picture of the origin of the various effects. The winners belong directly or indirectly to
The Sharvin-Sharvin experiment [Altshuler B.L. et al., JETP Lett. 35 (1982) 5881]: owing to the dependence of electron wave functions on the vector potential of a magnetic field (the Bohm-Aharanov effect), the conductivity of a long, thin-walled cylinder made of a normal metal is an oscillatory function of the magnetic flux which passes through the cylinder. The curve (thin line) calculated using the theory of B.L. Altshuler, A.G. Aronov and B.Z. Spivak [JETP Lett. 33 (1981) 94] for the magnetoresistance at liquid helium temperatures of a Li cylinder with length $10 \mathrm{~mm}$, diameter $1.1 \mu \mathrm{m}$, and wall thickness $0.12 \mu \mathrm{m}$ fits the experimetal curve (thick line) using only these parameters.

the great and famous Landau school of theoretical physics for which a clear physical understanding and interpretation is as important as exact formal results. Working in this spirit and with this approach, the prizewinners have togther made far reaching and stimulating discoveries while based in two different places, in Moscow and in St. Petersburg. It may well be possible that their work will eventually lead to novel and important types of electronic devices and sensors.

P. Wyder, Grenoble

\title{
FSU SMALL GRANTS PROGRAMME
}

\section{Supplementary Grants for EC\&E}

The American Physical Society (APS) decided last month to extend its small grants programme for direct aid to physicists in the former Soviet Union to include grants to east and central Europe (including the former Yugoslavia if conditions permit, and excluding Germany). The maximum grant is \$US 1000.- per individual and the funds must be used to supplement existing funds for FSU physicists to enable an existing collaboration with a group in E\&CE to be maintained after the physicists return home to the FSU.
To apply, group leaders in internationally recognized laboratories in E\&CE should submit by 15 April 1993 a proposal on behalf of FSU physicists to Irving Lerch, Director, International Affairs, APS, 335 East 45th St. New York, NY 10017, USA [tel./fax $+1-212$ 6827341 / 68725 32; apsap @ cunyvm]

The proposal should describe the collaboration, the facilities available to the FSU physicists, details of the physicists, and how the grant would be spent. A laboratory can apply for grants for up to four individuals.

\section{Programme Restructures}

The APS Small Grants Programme [see EN 23 (1992) 139] was set up in 1992 by an ad hoc APS task force on the crisis of basic research in the FSU. It aimed to provide SUS 500.- short-term survival mode grants directly to FSU physicists unable to travel or work abroad. Applications were channelled through a Moscow Coordination Committee (MCC) appointed by the task force. About \$US 900000.- has been distributed so far, mainly as small grants, but fellowships are also authorised. The MCC recommended in December that the programme be extended for a second year.

The MCC started last September to set up advisory committees based in the FSU republics to help it in its work; applications are processed by the committees before being forwarded to the task force. The MCC continues to certify that a laboratory has received funds so that matching funds provided, for example, by the Russian research minister can be sought (the Minister has also exempted grants from tax)

The MCC intends to enlarge its scope by becoming the Russian International Coordinating Committee on International Collaboration in Basic Science which will aim to obtain bilateral support for international research partnerships. The task force has been dissolved in the meantime, and grants are now approved by Bill Blanpied, a member (and immediate past-Chairman) of the APS Committee on International Scientific Affairs (CISA), who calls upon a network of advisors.

The task force's work has up to now been carried out by CISA operating from the APS secretariat. But with the increase in the volume of work and the need to move towards longer term and more stable forms of support, administration of the small grants programme will be taken over in mid- 1993 by the

\section{George Soros \\ Benefactor Extraordinaire}

George Soros grew up in Hungary and moved to London in 1947 to study at the London School of Economics. He worked as an investment manager, first in London and later in New York where he established the Quantum Fund - an investment fund reported to be now worth 7000 MSUS. The Open Society Fund, his first foundation, was set up in 1979 to support students and human rights activists in South Africa. It was followed in 1984 by a fund for his native Hungary that developed into a network of foundations for east and central Europe, some of which bear his name. Spin-offs include the Central European University, that gives graduate courses in Budapest and until next year in Prague, and the EastWest Management Institute that places managers for a short time in western companies.

International Science Foundation (ISA). This private body was established recently by George Soros (see box), the Hungarian financier, who made a widely reported commitment last December to donate 100 MSUS. ISA aims to support scientific culture in the FSU by offering grants, fellowships and endowments to both both individuals and institutions. E.W.A. Lingeman, Secretary of the EPS East-West Coordination Committee, who attended a CISA meeting in New York in January reports that ISA will soon launch an approximately 6 MSUS programme involving emergency six-month support for small research groups; a much larger Phase II programme should start in July 1993. An Executive Director and the Foundation's Board have not yet been formally appointed. 